\title{
Making psychedelics into medicines: The politics and paradoxes of medicalization
}

\author{
TEHSEEN NOORANI* \\ Department of Anthropology, Durham University, Durham, UK
}

(Received: April 30, 2019; accepted: July 10, 2019)

\begin{abstract}
This commentary considers efforts to turn psychedelics into medications that can be administered through healthcare systems as examples of "medicalization." I draw on ethnographic research both inside and outside of university-based clinical trials from 2014 to date, together with analogous examples from psychiatry and drug research and development. Rather than taking a normative stance on medicalization, I situate it in a wider political, economic, and cultural context to better understand its logics and effects. I begin by suggesting the resurgence of psychedelic science has been concerned with medicalization from the outset, recently prompting a crisis in the "psychedelics community" over its self-identity and values. Next, against the confident public messaging surrounding psychedelics, I consider how attempts to scale up and market psychedelic-assisted therapy could end up undermining the safety and efficacy of the therapy itself. I then outline the movements to decriminalize, legalize, and minimize the harms and risks of using psychedelics in their currently illicit therapeutic and recreational modalities. Finally, I explore how working toward psychedelic medicalization over the coming years may influence the movements toward decriminalizing and legalizing psychedelics use, focusing on the underarticulated ways in which medicalization may disregard or even hinder, rather than help, decriminalization and legalization efforts. I call attention to how the cost of gaining approval for therapies incentivizes the development of diluted-yet-profitable forms of psychedelic-assisted treatments, and how frameworks developed for "proper use" demarcate what counts as "abuse" and enable those with newly sanctioned access to psychedelics to condemn afresh their illicit use.
\end{abstract}

Keywords: psychedelics, clinical trials, medicalization, decriminalization, legalization, pharmakon

\section{INTRODUCTION}

"Health is not simply a cost to the nation to be reduced; contradictorily, it is also a market to be grown." (Dumit, 2012, p. 9)

Psychedelic medicine is undergoing rapid change. As clinical trial successes are publicized and mainstream media interest grows, the ground is fertile for pharmaceutical activity. This commentary piece was originally written for an audience of psychiatrists, therapists, and corporate and academic drug developers and researchers, on the topic of "turning psilocybin into a medicine" (Noorani, 2019). It draws on data from an ethnography of contemporary Anglo-American psychedelic science, and conversations within spaces where psychedelics are used and discussed in the US and UK. I situate the promise of psychedelic-assisted treatments in a broader analysis of scientific knowledge production and the politics of drug (ab)use, with a particular focus on two leading developments: psilocybin in the treatment of depressive disorders and 3,4-methylenedioxymethamphetamine (MDMA) in the treatment of post-traumatic stress disorder (PTSD; For the purposes of this commentary, MDMA is considered a psychedelic - not only as a cultural or a political entity, but also in terms of drug effects, I suggest that such a classification can be argued either way.).

\section{PHARMACEUTICALIZATION AND THE PSYCHEDELICS COMMUNITY}

The term "psychedelics community" is regularly deployed in order to claim injunctions and imperatives on behalf of an ecology of actors, including scientific researchers, shamans, indigenous healers, festival goers, therapists, drug policy and criminal justice reformers, psychonauts, recreational users, seekers, and artists who use, value, and wish to make more accessible altered states produced through psychedelics. Therefore, the term masks a heterogenous ensemble who shared a desire to defend their interests and practices from accusations of illegitimacy and harm since at least the Nixon-era crack-down on psychedelics use and research. In the past few years, the psychedelics community has faced an opposite challenge: to articulate its own vision in the face of the successful mainstreaming of psychedelics. The plethora of claims to universal truth found in contemporary psychedelics-using cultures, from scientists to indigenous practitioners and therapists to psychonauts, echo in politics of conviction, which deepen existing fractures between

\footnotetext{
* Corresponding address: Tehseen Noorani; Department of Anthropology, Durham University, South Road, Durham, DH1 3LE, UK; Phone: +44 191334 1612; E-mail: tehseen.n.noorani@ durham.ac.uk
}

This is an open-access article distributed under the terms of the Creative Commons Attribution-NonCommercial 4.0 International License, which permits unrestricted use, distribution, and reproduction in any medium for non-commercial purposes, provided the original author and source are credited, a link to the CC License is provided, and changes - if any - are indicated. 
those who take different stances on the advent of psychedelic pharmaceuticalization.

Once approved for medical use, psychedelic medicalizers hope to blend drug and psychotherapy in the form of psychedelic-assisted psychotherapy, a treatment modality novel for contemporary psychiatry. Medical historians have argued that the laboriousness and cost of psychedelic-assisted psychotherapy were reasons why such therapy could not compete with the emerging treatment paradigms of the 1950s, which entailed outpatient services and the selfadministered daily use of pharmaceutical drugs (Richert \& Dyck, 2019). Today, insistence by those who wish to develop scalable psychedelic-assisted therapies on the prioritization of safety, quality of service provision, and commitment to broad accessibility remains in tension with profit-oriented imperatives. At a structural level, competitive healthcare markets incentivize exaggerated claims to efficacy and produce conflicts of interest in knowledge production.

Over the past year, a deep disappointment and anger swelled up in the psychedelics community against the activities of Compass Pathways in particular and psychedelic for-profit enterprise in general, reflected in the Statement on Open Science and Open Praxis with Psilocybin, MDMA, and Similar Substances (Jesse, 2018). For many in the psychedelics community, these events were a wake-up call. Yet what has been alleged of Compass Pathways' practices - to streamline and simplify interventions, procure exclusivity rights and even where strategically advantageous, mislead interlocutors, advisors, and collaborators (Goldhill, 2018) - is not unusual within the pharmaceutical industry, revealing just how ill-matched that industry is with the collaborative traditions woven through the psychedelics community. Certainly, until this point, antagonisms existed within the psychedelics community. However, it was possible to argue that these actors were mostly more symbiotic than parasitic, with the terms of recognition and coexistence continually contested and redrawn. Without wanting to paint a harmonious picture of these groups and their interactions, with the ramping up of medicalization and the involvement of large amounts of capital, a new phase of psychedelic medicine is currently taking shape.

\section{MAKING A MEDICINE: MEDICALIZATION}

One way to approach the challenge of making psychedelics into a medicine is as an instance of what is called "medicalization" - the shaping of human problems as specifically medical in nature, in order that they can be studied, diagnosed, treated, and prevented as such (Conrad, 2007). We might ask, "why the medicalization of psychedelics now?" Certainly, the incidence of what have come to been called "deaths of despair" is rising, and effective pharmacological treatments are lacking. In addition, challenges to the claim that drugs are fixes for "chemical imbalances" (Moncrieff, 2007) are gaining traction (e.g., https://www. madinamerica.com), and pharmaceutical company profits are threatened by the lack of novel psychotropic drugs (Friedman, 2013). With a proliferation of psychometric, epidemiological and in vivo brain imaging data, and research teams at well-regarded universities, psychedelic science is poised to answer pharma's call, retooled, rebooted-and-suited for the development of cutting-edge drug-assisted therapeutics (Langlitz, 2013).

Histories of the pharmaceutical industry suggest a bad track record of medicalization when it comes to psychiatric problems, what a host of service user, psychiatric survivor, mad pride activists, and critical psychiatrists have called "problems in living" (Szasz, 1961). For example, the psychiatric medicalization of depression has been a tale of biochemical and individualizing responses to problems elsewhere understood in terms of systemic issues of disconnection, alienation, and isolation, which require collective interventions (for instance, Horwitz \& Wakefield, 2007). And yet, it is tempting to give in to a culturally ingrained epochalism that "this time is different." Progressivist stories of increased understanding, including increased drug targeting, are used to steel political zeal.

The resurgence of the applied science of psychedelics has in fact always been oriented toward medicalization, seeking application in areas such as addiction, palliative care, obsessive-compulsive disorder, PTSD, and social anxiety. Psychedelics are commonly referred to as "tools," inviting their mastery and a sense of automaticity, suggesting the effects of clinic-based psychedelic administration follow a linear causative model typical of pharmaceutical explanation. The mystical experience has in recent years been modeled, quantified, and coveted as the key mediating variable in the successful treatment of therapeutic targets (e.g., Griffiths et al., 2011). Referred to in overlapping ways as ego-dissolution, ego-death, peak, or corrective emotional experiences, it has been reified, homogenized, and charged with the power to produce lasting change in people, on the model of the "cure" so seductive to medical discourse. "Integration" is the catch-all term for a range of ways of understanding the work required to bring the meaning and fruits of the psychedelic experience to bear upon one's life in the aftermath of the acute drug effects.

Yet, there are stark discontinuities between the discrete, point-like, enduring changes reported in the clinical research literature and the experiences that have been described to me by trial participants and guides, underground therapists and psychonauts, for whom integration is tricky and ongoing work. The Senior Study Guide at the Johns Hopkins Psychedelics Research team, Mary Cosimano, actively encourages participants to seek out community-based support to help with integration, noting that the integration of the themes and topics that arise from a psychedelic session continues well into 6 months and even the 1 year (final) follow-up meetings with participants (personal communication, June 23, 2019). Such community-based support includes a spate of community-based psychedelics societies and psychedelic integration groups that have sprung up, since the resumption of clinical trials in the US over the past two decades.

It is common at integration groups and psychedelic society meetings I have attended to meet ex-trial participants looking for more guidance and ways to extend and scaffold their psychedelic experiences. I find it productive to think about integration alongside the Greek notion of the drug as pharmakon - both poison and cure. In contrast with the toxicological goal of clearly distinguishing poison and cure 
by dose, psychedelic experiences appear to re-present the logic of both poison and cure - both new solutions and new problems, generative riddles that require further work to unknot.

Clinical trial data tend to ignore these new problems and riddles that emerge from participant experiences when these are unrelated to the clinical targets the trials were designed to treat. Indeed, assessing the outcomes of psychedelic experiences only on the basis of participant experiences within the research trial setting reveals little about the ongoing community-based containers that ex-trial participants participate in, and themselves build, to successfully navigate new journeys initiated by psychedelic experiences. Yet, parsing out these phenomena and their relationship to differential levels of community support is in principle possible, revealing avenues for participatory and ecologically minded forms of scientific knowledge generation, methodologies thus far deprioritized in the psychedelic science renaissance.

In developing psychedelic therapy at scale, several points are worth considering. First, the time commonly allocated to integration in clinical trials is costly and, without a fuller account of when and how it is done well, is liable to be attenuated and/or automated in for-profit contexts. Second, introducing psychedelics into a medical culture that has long-promised the targeted use of magic bullets for mental distress will only heighten the disappointment when the experiences or effects are not as straightforward as had been hoped for, especially for people for whom other treatments have failed. Third, psychedelics can produce new dissociations that need grounding. A topic I have often encountered in integration groups and psychedelic society meetings is how "coming down" after a particularly strong psychedelic experience is less coming back than arriving somewhere else, fresh with the experience of clarity, purity and/or simplicity, only to be thrust back into the intractable cruelties of mundanity, and everyday systemic injustice: the rat race, social alienation, discrimination, and so on. Indeed, in a for-profit system, a perverse incentive exists to ensure that further treatment is needed, capitalizing on the pharmakonlike properties of psychedelic experiences even while marketing the intervention as producing discrete, lasting, beneficial changes.

Regulators are also playing a key role in shaping the scientific knowledge production. Justified in terms of the large estimated "unmet need," the US Food and Drug Administration (FDA) and the European Medicines Agency has recently approved phase three trials using psilocybin to treat depressive disorders and MDMA to treat PTSD. In order to approve legal use, these regulators require that randomized controlled trial (RCT) data - considered the gold-standard of evidence - demonstrate "safety" and "efficacy" in treatment. The focus on acquiring the RCT data necessary for drug approval sidelines other kinds of research questions and methods, including qualitative research, research to explore mechanisms of change, and research into the effects of the context of the drug's administration, even as clear failures of blinding continue to frustrate the RCT paradigm itself (cf. Hendy, 2018).

Regulators require that the provision of new medications be scalable if they are to green-light late-stage clinical trials.
Given the size of the potential markets in the cases of depressive disorders and PTSD, viable companies are currently planning the rapid training of thousands of psychedelic therapists. Yet, the role of guides or therapists may be far more complex and less automatizable than often suggested in the contemporary clinical literature, including through the depiction of their function in peer-reviewed journal articles that demand short methodology sections and continue to emphasize the drug effects. Debates across the psychedelics community continue over whether guides or therapists should have personal experience of psychedelics, and how such figures best learn how to act - and not act - at the right times (e.g., Gasser, 2017). Few examples of useful guide interventions can also be found in the limited qualitative research that has been undertaken (e.g., Noorani, Garcia-Romeu, Swift, Griffiths, \& Johnson, 2018). The prospect of a new hierarchy of formally credentialed versus uncredentialed therapists has been regarded within the psychedelics movement with irony among both those who extoll anti-hierarchy insights gained from the psychedelic experience and long-standing and respected guides who are skeptical of such endeavors as only increasing transference (e.g., Feldmar, 2015) - including attribution to the therapist of the power to heal.

There remain pressing questions about whether marginalized groups will be able to access the provision of medicalized psychedelic therapy. This will be shaped by the structure of the wider healthcare system and the actuarial strategies involved in calculating insurance policies. Meanwhile, clinical trials are generating path dependencies in the form of "standardized protocols and procedures" based upon the typical participant to date - the White, educated, middle-classed subject who can afford to take the time required for all the meetings and paperwork (see Michaels, Purdon, Collins, \& Williams, 2018). It could be argued that in early-stage trials, researchers are operating on tight budgets and do not have the luxury of prioritizing diversity among participants, while advanced-stage trials mark the time to demonstrate efficacy and no new safety concerns rather than altering trial procedures and protocols. And yet, the knowledge generated may consequently only apply to a privileged minority, and down the road, use in alternative ways could continue to be considered as mis-use, feeding litigation, and moralism (see section "Medicalization and decriminalization: Troubling the 'Both-And"'). Recently, MAPS collaborated with Monnica Williams to set up a Phase III research site prioritizing the enrollment of a nonnormative population - including those affected by "racebased trauma" (Williams \& Leins, 2016) - and Williams has since listed the myriad barriers to success that were experienced before the site closed (see Watson, 2018).

\section{ALREADY A MEDICINE: DECRIMINALIZATION}

To date, I have sketched ways in which medicalization presents challenges, including ways that the very attempt to medicalize at scale may change that which it is medicalizing. These are some dangers of putting all eggs in the medicalization basket. What is often overlooked in discussions of this sort is how psychedelics are already medicines 
for many - indeed, the question at hand more accurately concerns turning them into medications - what regulators term "drug products" - to be sold by pharmaceutical companies.

In Northern European and American contexts, psychedelics use has been widespread across underground, indigenous, and sacramental contexts, continuing in underground contexts even after their overground use was banned at the end of the 1960s. This includes spiritual and therapeutic use, exploratory scientific inquiry, community-based harmreduction activities, and the building of a diverse array of containers for holding and integrating psychedelic experiences, including the aforementioned integration groups. For the non-sanctioned use of psychedelic medicines, however, criminalization itself exacerbates and produces new harms. Community-based harm reduction has thus been central, including holding safe spaces, making drug testing available, disseminating information on safely growing, storing, extracting and propagating psychedelic substances, and educating law enforcement.

The stakes are even higher for those, such as the UK-based Release and US-based Drug Policy Alliance, who situate psychedelic drug within wider ecologies of illicit drug use. For these organizations, legalizing all drugs and focusing on harm reduction would be a big stepping stone to ending the War on Drugs, a key driver behind the growing prison-industrial complex, racist criminal justice system, and militaristic global interventionism. Public support for decriminalization and legalization is becoming an increasingly politically expedient goal, as models of drug decriminalization and legalization proliferate across the world, scientists, and analysts highlight the absurdity of current drug laws (e.g., The Beckley Foundation, 2016), bipartisan concerns builds around the size of prison industries, and there is a greater framing of drug addiction as a public health issue. After recent community-driven, noncorporate successes in reducing criminalization in Denver and Oakland, decriminalization advocates must now show that the psychedelics community can address the risks of psychedelics effectively. MAPS' Zendo project is a good example of this kind of work, from an organization nevertheless committed to medicalization.

However, for criminal justice and drug policy reform advocates in the psychedelics community, it is essential that the complexity and diversity of innovative and communitybased ways of regulating drugs and people in illicit contexts not be carpeted over by regulations designed and driven by the interests of single actors and large capital. This has occurred with much of the marijuana industry in the US, particularly in jurisdictions that ignore calls for "equity" provisions in policy and legislation as a response to the ongoing impact of the War on Drugs.

One obstacle to decriminalizing and legalizing all drugs along the lines proposed by Release is a salient "psychedelic exceptionalism." The claim is that psychedelics (and marijuana) are different from other drugs, or that they are not drugs at all, and that psychedelics users are not like "other drug users," despite the fact that psychedelics are commonly used alongside other illegal drugs - drugs which are perceived to be used in more racially stratified ways than they in fact are. In addition, ideas of set and setting, as well as purity of drug and mode of administration, seem to really matter for understanding the adverse effects of all drugs. Experiences and effects are fundamentally shaped by the immediate context of use, resources (e.g., consider the privilege of psychedelic clinical trial participants noted above), surveillance, and policing (Hart, 2013).

\section{MEDICALIZATION AND DECRIMINALIZATION: TROUBLING THE "BOTH-AND"}

What is the relationship between the medicalization of psychedelics and their decriminalization and legalization? While medicalization efforts may trouble some of the myths about these drugs, like that they are inherently dangerous, the importance of challenging the myths lies not (or not only) in rescuing the reputation of the drugs, but in challenging the harms against those demonized along with them. The US state-legal medical marijuana programs only led to minor and mostly superficial changes in criminal justice policy. Even following full legalization for adult use, arrests across many states remained disproportionately high for Black and Hispanic populations (Drug Policy Alliance, 2018). Thus, it is logical to suggest that those seeking the wider availability of psychedelic medicines should pursue both medicalization and decriminalization. In the remainder of this commentary, I highlight some challenges in taking this both-and approach.

In providing frameworks for "proper use," medicalization delineates the conditions of "abuse." Clinical trials are generating findings with no wider ecological validity than the highly demarcated use of a highly rarified grade of a specific psychedelic substance. The doxa, oft-rehearsed across the psychedelics community, of "drug-set-setting" can be mobilized in support of creating a bifurcated schedule, where "drug products" are scheduled separately from "substances," despite entailing identical chemicals. Not anticipated by the US Controlled Substances Act, such bifurcations have been created on an ad hoc basis, for cannabinoids, gamma hydroxybutyrate, and ketamine (Ismail Lourido Ali, personal communication, June 19, 2019), offering the FDA a legal mechanism by which to create markets for tightly regulating licit use while avoiding concerns with or backlash from rescheduling substances in toto.

The creation of bifurcated schedules for psychedelics is of great concern for those in the psychedelics community who seek to end the criminalization of psychedelics use in general, because with bifurcated schedules, psychedelics use outside of sanctioned spaces will remain criminalized. The position of not condoning the unsupervised use of psychedelics, adopted by a range of influential psychedelic scientists, has the potential to take on renewed significance, cleaving medicalized "treatments" from a vast ecology of social and cultural uses of plant-based and synthetic psychedelics on the basis not of research into the relative efficacy of these approaches, but of the need and ability to recoup costs for RCTs and offer business plans that satisfy regulators. That is, the division between the good psychedelics user and the bad psychedelics abuser can be justified as cautiously "sticking to the science." 
In an ironic aside, any pro-medicalization message that non-clinical use of psychedelics is dangerous may be selffulfilling by producing more anxious or paranoid mindsets and morally punitive cultural settings for their use. This is the realizing of psychedelic exceptionalism in an even tighter form.

In the latest example of bifurcated scheduling, in 2019, the FDA-approved Spravato, Janssen's esketamine nasal inhaler, for use in low doses under medical supervision to treat treatment-resistant depression. In contrast with the currently existing off-label use of high doses of intravenously administered ketamine, which appears to show great promise (Zarate \& Niciu, 2015), only one of the three shortterm RCTs with the nasal spray revealed a statistically significant reduction in participants' depression scores (Food and Drug Administration, 2019). Moreover, participants with suicidal ideation were screened out of the trials and the reductions in the one successful short-term trial were clinically small. Yet, this single trial has paved the way for a potential blockbuster drug product for Janssen's parent pharmaceutical company (Ryan, 2019).

In psychedelic-assisted psychotherapy, where the time and labor accorded preparation, guiding and integration suggest effectiveness and cost are correlated, the case of Spravato reminds us of the incentive to gain approval for diluted forms of the intervention by attenuating the protocol, therapist training, and so forth. If the pursuit of medicalization leads to bifurcated scheduling and the approval of diluted therapeutic forms, a new choice may emerge to those seeking treatment: relatively ineffective but legal psychedelic therapy or more effective but criminalized underground psychedelic therapy. Meanwhile, we may expect the high financial stakes involved in licit use to produce the kinds of interminable debates over (in)efficacy that plague approved antidepressants (e.g., Hengartner, 2019).

Finally, with the institutionalization of access to previously illicit drugs for more powerful demographics, decriminalization for others may be deprioritized, especially when financial profit or self-legitimation is at stake. For instance, even though the prescription drugs Adderall and Ritalin are almost interchangeable with the illegal drug methamphetamine (Hart, 2013), among those with access to prescriptions, their families, and care givers, there is little sympathy for the injustice faced by those using the near-same drugs illegally. In the US, scheduling and sentencing are not aligned, as illustrated by the severe criminalization of schedule 2 and 3 drugs including methamphetamine and cocaine. This makes community-based resources for decriminalization and harm reduction all the more important, because medicalization will not automatically lead to either outside of licit use. A young person of color-taking mushrooms or MDMA illegally at a rave or festival may in fact come to embody the irresponsible "other" to clinic-based psychedelic therapy.

\section{CONCLUSIONS}

It is an aptly psychedelic insight to emphasize the context the wider "set" and "setting" - of psychedelic medicalization. This includes the role of community-based support in integrating psychedelic experiences that are bracketed aside by narrowly constructed RCTs, and decriminalization and harm-reduction efforts that exist alongside - and perhaps even in spite of - calls for medicalization. As I have indicated, medicalization can occur in many ways, ranging from faster and more profit-driven to slower and more participatory, from embedding a "right way" to use psychedelics, to ensuring regulators and the public more deeply engage an open-ended plurality of safe ways of using psychedelics (These present opportunities for emerging multidisciplinary research into psychedelics, such as the newly established Centre for Psychedelic Research at Imperial College London, to take up.). However, large capitalbacked efforts at medicalization are increasingly dominating public discourses, marshalling resources to shape broader understandings of psychedelics, both directly and indirectly via the way the scientific research is designed - as the pharmaceutical industry has done regarding previous drugbased interventions for psychiatric problems.

One frustration for some in the psychedelics community is that the scaling psychedelic-assisted psychotherapy for depression or PTSD is an opportunity, again, to ask collective questions about the increasing prevalence of mental illness in our societies, instead of medicalizing and treating individuals. In the case of depression, for example, we might more astutely consider the economic, cultural, and epistemic forces that are converging to make psychedelics "the" new solution for depression. We may also seek out ways of ensuring greater meaningful social connection, the embrace of difference, community spaces, non-precarious employment, and affordable housing - all phenomena that underlie many such problems in living. However, it appears that such challenges are being relegated to a countercultural and somewhat nostalgic politics, where the psychedelic experience enables the revisioning of societal paradigms. Instead of psychedelicizing pharma, the pharmaceuticalizing of psychedelics hopes to harness the transformative power of psychedelics "one individual at a time."

And in so doing, medicalization may be a kind of a social pharmakon. In promising to make psychedelic experiences more available, we could say it reduces the "plasticity" (Carhart-Harris \& Nutt, 2017) of these experiences - how they are understood and held and who gets to have them even if in the hope that once medicalized, a truly diverse ecology of psychedelic-assisted therapies will flourish. However, lessons from the history of the pharmaceutical industry, alongside the ongoing harms of the War on Drugs, press us to be cautious. The pharmaceuticalization of psychedelic therapy is gaining momentum. It is tempting to ask if the psychedelics community is ready, but to do so may be to presume a single subject position that, increasingly, no longer holds.

Acknowledgements: The author would like to thank Ismail Lourido Ali and Jeffrey Guss for comments and conversation that helped shape this article.

Conflict of interest: The author declares no conflict of interest. 


\section{REFERENCES}

Carhart-Harris, R. L., \& Nutt, D. J. (2017). Serotonin and brain function: A tale of two receptors. Journal of Psychopharmacology, 31(9), 1091-1120. doi:10.1177/0269881117725915

Conrad, P. (2007). The medicalization of society: On the transformation of human conditions into treatable disorders. Baltimore, MD: Johns Hopkins University Press.

Drug Policy Alliance. (2018). From prohibition to progress: A status report on marijuana legalization (Report). Retrieved from http://www.drugpolicy.org/sites/default/files/dpa_marijuana_ legalization_report_feb14_2018_0.pdf

Dumit, J. (2012). Drugs for life: How pharmaceutical companies define our health. Durham, NC: Duke University Press.

Feldmar, A. (2015). Psychedelic therapy: Notes from the underground [Conference presentation]. Breaking Convention. Retrieved from https://vimeo.com/136504202

Food and Drug Administration. (2019). Briefing document for psychopharmacologic drugs. Paper presented at Psychopharmacologic Drugs Advisory Committee and Drug Safety and Risk Management Advisory Committee Meeting. Retrieved from https://www.fda.gov/media/121376/download

Friedman, R. A. (2013). A dry pipeline for psychiatric drugs. The New York Times. Retrieved from https://www.nytimes.com/ 2013/08/20/health/a-dry-pipeline-for-psychiatric-drugs.html

Gasser, P. (2017). Psychedelic psychotherapy in Switzerland [Conference presentation]. Nätverket för psykedelisk vetenskap. Retrieved from https://www.youtube.com/watch?v= rDFbMUdB_pU

Griffiths, R. R., Johnson, M. W., Richards, W. A., Richards, B. D., McCann, U., \& Jesse, R. (2011). Psilocybin occasioned mysticaltype experiences: Immediate and persisting dose-related effects. Psychopharmacology, 218(4), 649-665. doi:10.1007/s00213011-2358-5

Goldhill, O. (2018). A millionaire couple is threatening to create a magic mushroom monopoly. Quartz. Retrieved from https://qz. com/1454785/a-millionaire-couple-is-threatening-to-create-amagic-mushroom-monopoly/

Hart, C. (2013). High price: A neuroscientist's journey of selfdiscovery that challenges everything you know about drugs and society. New York, NY: HarperCollins Publishers.

Hendy, K. (2018). Placebo problems: Boundary work in the psychedelic science renaissance. In B. C. Labate \& C. Cavnar (Eds.), Plant medicines, healing and psychedelic science: Cultural perspectives (pp. 151-166). New York, NY: Springer.

Hengartner, M. P. (2019). Scientific debate instead of beef: Challenging misleading arguments about the efficacy of antidepressants. Acta Neuropsychiatrica, 31(4), 235-236. doi:10.1017/neu.2019.23
Horwitz, A. V., \& Wakefield, J. C. (2007). The loss of sadness: How psychiatry transformed normal sorrow into depressive disorder. Oxford, UK: Oxford University Press.

Jesse, R. (2018). Statement on open science and open praxis with psilocybin, MDMA, and similar substances. Open Letter. Retrieved from https://files.csp.org/open.pdf

Langlitz, N. (2013). Neuropsychedelia: The revival of hallucinogen research since the decade of the brain. Berkeley, CA: University of California Press.

Michaels, T., Purdon, J., Collins, A., \& Williams, M. (2018). Inclusion of people of color in psychedelic-assisted psychotherapy: A review of the literature. BMC Psychiatry, 18(245), 1-9. doi:10.1186/s12888-018-1824-6

Moncrieff, J. (2007). The myth of the chemical cure: A critique of psychiatric drug treatment. New York, NY: Palgrave Macmillan.

Noorani, T. (2019). Of fetishes and pharmaka: Paradoxes of psychedelic medicalization [Conference presentation]. In Making psilocybin into a medicine: A multidisciplinary approach Conference. London: King's College London.

Noorani, T., Garcia-Romeu, A., Swift, T. C., Griffiths, R. R., \& Johnson, M. W. (2018). Psychedelic therapy for smoking cessation: Qualitative analysis of participant accounts. Journal of Psychopharmacology, 32(7), 756-769. doi:10.1177/026988 1118780612

Richert, L., \& Dyck, E. (2019). Psychedelic crossings: American mental health and LSD in the seventies. Medical Humanities. Advance online publication. doi:10.1136/medhum-2018011593

Ryan, J. (2019). Bait and switch: The great ketamine "breakthrough". Rxisk. Retrieved from https://rxisk.org/bait-andswitch-the-great-ketamine-breakthrough/

Szasz, T. (1961). The myth of mental illness: Foundations of a theory of personal conduct. New York, NY: Harper \& Row.

The Beckley Foundation. (2016). Out of UNGASS: A new approach. Public Letter. Retrieved from https://beckleyfoundation. org/wp-content/uploads/2016/05/Public-Letter-2016.pdf

Watson, E. C. (2018). Black Americans are building a space in psychedelic drug culture after being ignored for decades. OkayPlayer. Retrieved from https://www.okayplayer.com/ originals/black-people-psychedelic-drugs-monnica-williams. html

Williams, M. T., \& Leins, C. (2016). Race-based trauma: The challenge and promise of MDMA-assisted psychotherapy. Multidisciplinary Association for Psychedelic Studies (MAPS) Bulletin, 26(1), 32-37.

Zarate, C. A., \& Niciu, M. J. (2015). Ketamine for depression: Evidence, challenges and promise. World Psychiatry, 14(3), 348-350. doi:10.1002/wps.20269 